\title{
Aquatic Therapy- A Boon to Rehabilitation
}

\author{
Arnaaz Rusi* \\ Department of Musculoskeletal and Sports, University, AquaCentric, Andheri, India \\ *Corresponding Author: Arnaaz Rusi, Department of Musculoskeletal and \\ Sports, AquaCentric, Andheri, India.
}

Received: February 07, 2020

Published: March 01, 2020

(C) All rights are reserved by Arnaaz Rusi.
Aquatic therapy refers to exercises conducted in a warm therapeutic pool. Warmth of the water helps to relax the muscles and in pain reduction. while performing the exercises. Treatments and exercises are performed while floating, partially submerged, or fully submerged in water. When the water is at the level of shoulder, the body weight is reduced by $90 \%$. Due to which there is minimal compressive forces on the joints and spine as there is no gravity in water. This property of water is beneficial as the exercises conducted become more feasible for the patient. The beauty of water is its hydrostatic pressure, which is present on all sides which gives resistance therefore it helps in strengthening of muscles. It also helps to improve cardiovascular endurance. Walking, running or cycling is much easier in water due to weightlessness. Another property of water is Viscosity which allows a gentle and progressive resistance to exercises.

Aquatic therapy can be used for orthopaedic and sports, neurological and women's health. In orthopaedic conditions when land based conventional rehabilitation is difficulty aquatic therapy is more convenient and has rapid progression. It is widely used postoperative cases where buoyancy helps to get the desired range of motion with minimal pain and discomfort. In patients post fractures where weight bearing is restricted, this therapy can be started to prevent immobilisation and muscle tightness. Rehabilitation can be started at an early stage which helps to progress the prognosis.

In neurological conditions patients who are wheelchair bound can stand and walk in water which helps in gait training and builds up their confidence. Exercises in water puts less strain on their body and they are able to perform exercises for a longer time. It also helps to reduce spasticity and also provides sensory integration. Due to hydrostatic pressure core and trunk stability can be achieved. Ai Chi is a form of aquatic therapy used for relaxation as well as balance training.

Watsu technique of aquatic therapy is used for relaxation in Antenatal and post natal cases. Many metabolic conditions like thyroid, PCOS, are becoming common due to unhealthy life style. Aquatic therapy helps in managing them by improving fitness by strengthening and endurance training.
Aquatic therapy can be used for any age group, for geriatric population it is beneficial as there are no chances of fall or injury in water making it safe for them to exercise.

For pediatric population it is more like a play therapy for them therefore they are more enthusiastic and looking forward for therapy. The property of hydrostatic pressure gives constant sensory awareness which helps in sensory integration techniques in special children. It enhances the therapy as it is not painful and the child is more co-operative.

In field of sports, aquatic therapy accelerates the recovery from the injury making the player return to sports. The rehabilitation can be started early which prevents muscle wasting and joint stiffness..

The use of water for therapeutic purposes first dates back to 2400 B.C. in the form of hydrotherapy, with records suggesting that ancient Egyptian, Assyrian, and Mohammedan cultures utilized mineral waters which were thought to have curative properties through the 18th century.

Now emergence of Aquatic therapy has taken place with evolution of development of modern techniques including the Halliwick Concept and the Bad Ragaz Ring Method which have given new dimension to the field of rehabilitation.

\section{Assets from publication with us}

- Prompt Acknowledgement after receiving the article

- Thorough Double blinded peer review

- Rapid Publication

- Issue of Publication Certificate

- High visibility of your Published work

Website: https://www.actascientific.com/

Submit Article: https://www.actascientific.com/submission.php Email us: editor@actascientific.com

Contact us: +919182824667 